Boise State University

ScholarWorks

8-16-2010

\title{
2D Ground-Penetrating Radar AVO Response to a 3D Dielectric Permittivity Anomaly
}

John H. Bradford

Boise State University

(C2010 IEEE. Personal use of this material is permitted. However, permission to reprint/republish this material for advertising or promotional purposes or for creating new collective works for resale or redistribution to servers or lists, or to reuse any copyrighted component of this work in other works must be obtained from the IEEE. DOI: 10.1109/ICGPR.2010.5550196 


\title{
2D ground-penetrating radar AVO response to a 3D dielectric permittivity anomaly
}

\author{
John H. Bradford \\ Dept. of Geosciences \\ Boise State University \\ Boise, Idaho, USA \\ johnb@cgiss.boisestate.edu
}

\begin{abstract}
To evaluate the amplitude vs offset response of GPR to small distributions of hydrocarbon contamination, I acquired multi-offset $450 \mathrm{MHz}$ GPR data in TE and TM modes over a buried rectangular tank filled with gasoline saturated sand. All dimensions of the tank were less than one wavelength at the characteristic antenna frequency. The permittivity ratio at the moist sand/gasoline sand boundary, estimated by fitting the Fresnel equations to the observed amplitudes and by Brewster's Angle analysis, differed from that obtained through migration velocity analysis by no more than 12\%. 2D FDTD modeling reproduced amplitude characteristics for 3 o f 4 target/polarization combinations and explained some deviations from the Fresnel curves. Additional deviations may be caused by out-of-plane polarization effects or heterogeneity not included in the 2D model.
\end{abstract}

Keywords-AVO, NAPL, $3 D$

\section{INTRODUCTION}

In this study, I focus on the application of amplitude variation with offset (AVO) analysis for estimating the permittivity ratio at a reflecting boundary. For electromagnetic (EM) wave propagation, the AVO response depends strongly on the polarity of the electric field, and the contrast of permittivity (e) and conductivity (o) between the incident and reflecting medium [1]. Many light NAPL (LNAPL) and dense NAPL (DNAPL) contaminants have much lower e and o than water $[2,3]$ so that the introduction of a contaminant at depth (i.e. a migrating LNAPL plume, or DNAPL pool at the base of an unconfined aquifer) can lead to a dramatic drop in e and o as the contaminant displaces water in the pore space. This is an anomalous scenario and, when the NAPL rich zone has sharp boundaries relative to the signal wavelength, the NAPL is suitable for AVO investigation.

When carefully calibrated with a minimal amount of borehole information, GPR imaging integrated with AVO analysis can yield a rich source of data that includes geometric relationships and detailed material property information. This can significantly improve the ability to predict the location of isolated contaminant bodies. Additionally, since AVO analysis depends only on energy reflected from the upper surface of a target boundary, it is possible to determine the permittivity of the zone just below the boundary, even if no energy penetrates the boundary. While GPR AVO analysis remains a relatively immature area of study, several authors have investigated its potential as a characterization tool. Bergmann et al. [4] briefly discuss AVO response in the context of a broader synthetic case study. Reppert and Morgan [5] use Brewster's angle to determine the permittivity contrast at a reflecting boundary. Baker [6] presents a modeling study indicating potential for detecting LNAPLs based on the transverse AVO response. Zeng et al. [1] present a detailed modeling study considering the effects of varying the colecole parameters on the AVO response. More recently, Bradford and Deeds [7] discussed field results showing that reliable permittivity estimates can be obtained from AVO analysis of thinbed reflections associated with NAPL contamination. In this study, I discuss the results of a physical model designed to evaluate the GPR AVO response to a subwavelength $3 \mathrm{D}$ permittivity anomaly.

\section{PHYSICAL MODEL}

\section{A. Data acquisition}

The physical model was constructed by first filling two thin-walled plastic containers $(0.46 \mathrm{~m} \times 0.30 \mathrm{~m} \times 0.41 \mathrm{~m})$ with washed, coarse-grained, quartz sand, then saturated one container with water and the other with gasoline. The two containers were buried adjacent to each other at a depth of 0.53 $\mathrm{m}$ in a test pit filled with the same quartz sand. The long axis was oriented parallel to the survey direction and the narrowest dimension was oriented vertically simulating a $0.30 \mathrm{~m}$ thick LNAPL layer (Figure 1). The water table in the pit was maintained at a depth of $0.61 \mathrm{~m}$, which is about $0.08 \mathrm{~m}$ below the top of the containers. This simulated a floating LNAPL layer depressing the water saturated zone so that about $74 \%$ of the LNAPL thickness lies below the water table. 


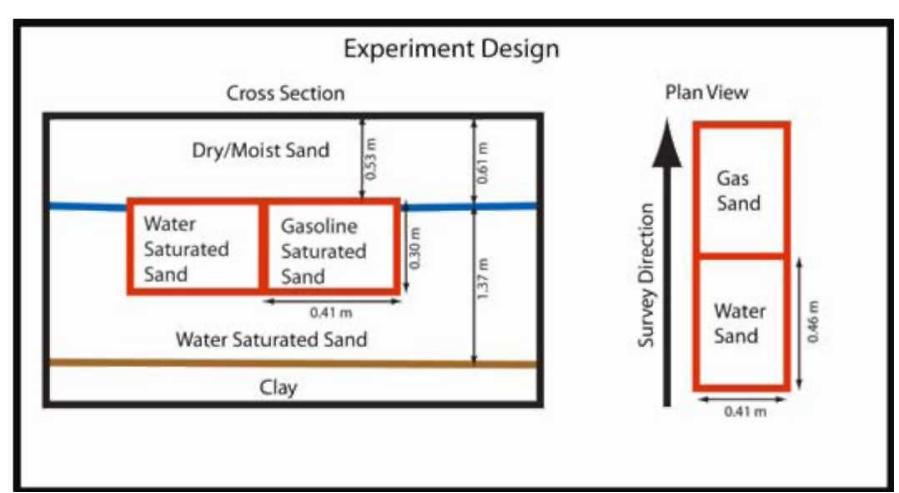

Figure 1. Schematic of the LNAPL AVO experiment (not to scale). The boundaries of the test pit were a minimum of $2 \mathrm{~m}$ from the survey area and did not interfere with the experiment.

Two, $1.2 \mathrm{~m}$ long CMP surveys were acquired with a Sensor's and SoftwareTM PulseEkko 1000 system using 450 Mhz antennas. The first survey was acquired in PE mode (field polarized parallel to image plane), with $0.025 \mathrm{~m} \mathrm{CMP} \mathrm{spacing,}$ $0.050 \mathrm{~m}$ offset increments, a minimum offset of $0.28 \mathrm{~m}$ (antenna center-to-center distance) and 30 traces/CMP giving a maximum offset of $1.73 \mathrm{~m}$. The second survey was acquired in the TE mode (field polarized perpendicular to the image plane), with a $0.050 \mathrm{~m}$ CMP spacing, $0.050 \mathrm{~m}$ offset increments, a minimum offset of $0.20 \mathrm{~m}$, and 32 traces/CMP giving a maximum offset of $1.75 \mathrm{~m}$. NMO corrected CMP gathers are shown in Figure 2.

\section{B. Velocity analysis}

For the physical model in this discussion, I expect the velocity to change by as much as a factor of 3 , both laterally and vertically, as the wave travels from the NAPL saturated sand to moist or water saturated sand. Under these conditions, conventional NMO velocity analysis fails and accurate imaging requires pre-stack depth migration (PSDM) with velocities obtained from inversion or migration velocity analysis. Prestack depth migration velocity analysis with a layer stripping approach yielded the velocity model shown in Figure 3A. Prestack depth migration with this velocity model places reflections from the containers at the correct spatial position (Figure 3B).

The PSDM velocity model can be used to estimate the permittivity ratio at the boundaries between the vadose zone and water saturated sand and gasoline saturated sand containers. For the sand just above the containers, our analysis gives a velocity of $0.116 \mathrm{~m} / \mathrm{ns}$. The permittivity ratio at the water sand boundary is $\varepsilon_{2} / \varepsilon_{1}=(0.116 \mathrm{~m} / \mathrm{ns} / 0.058 \mathrm{~m} / \mathrm{ns})^{2}=$

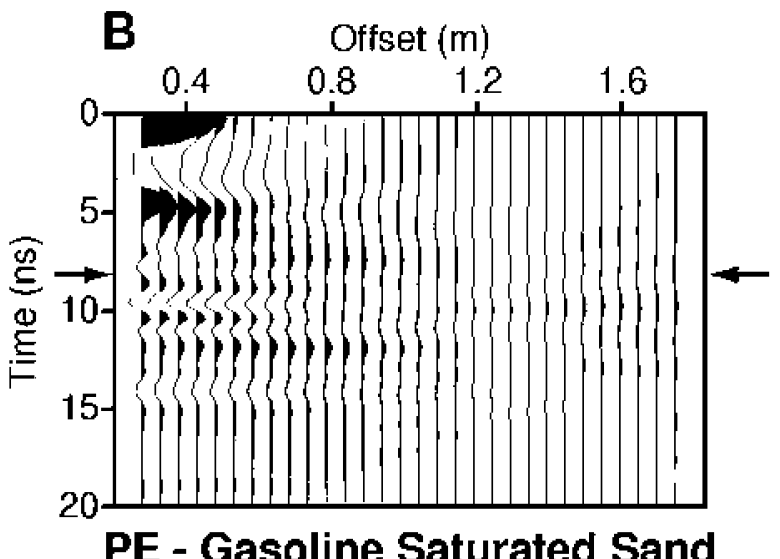

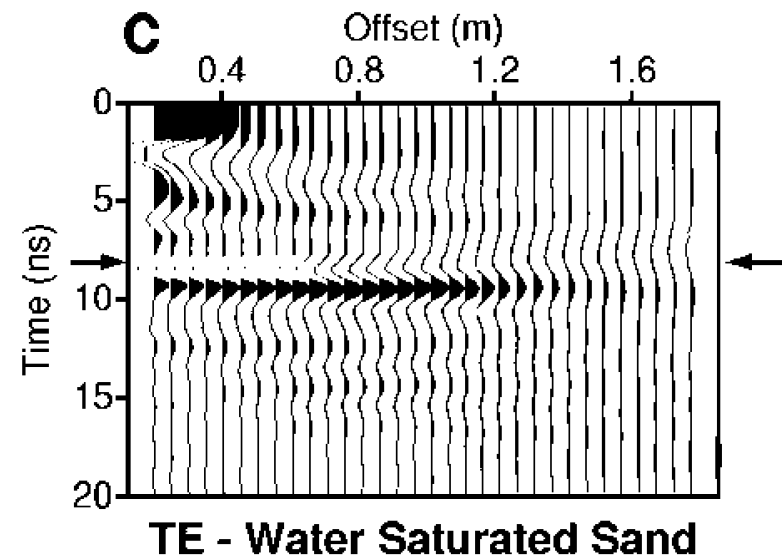

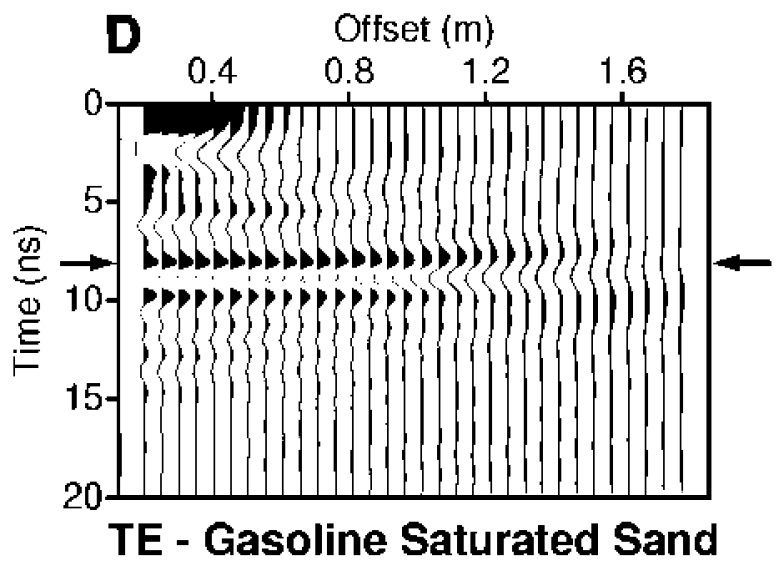

Figure 2. NMO corrected CMP supergathers. Arrows indicate the onset of top-of-container reflections. $8_{\mathrm{B}}$ is clearly evident in the $\mathrm{PE}$ gasoline sand data (B). Offsets were not large enough to observe $8_{\mathrm{B}}$ for the water sand reflection. 

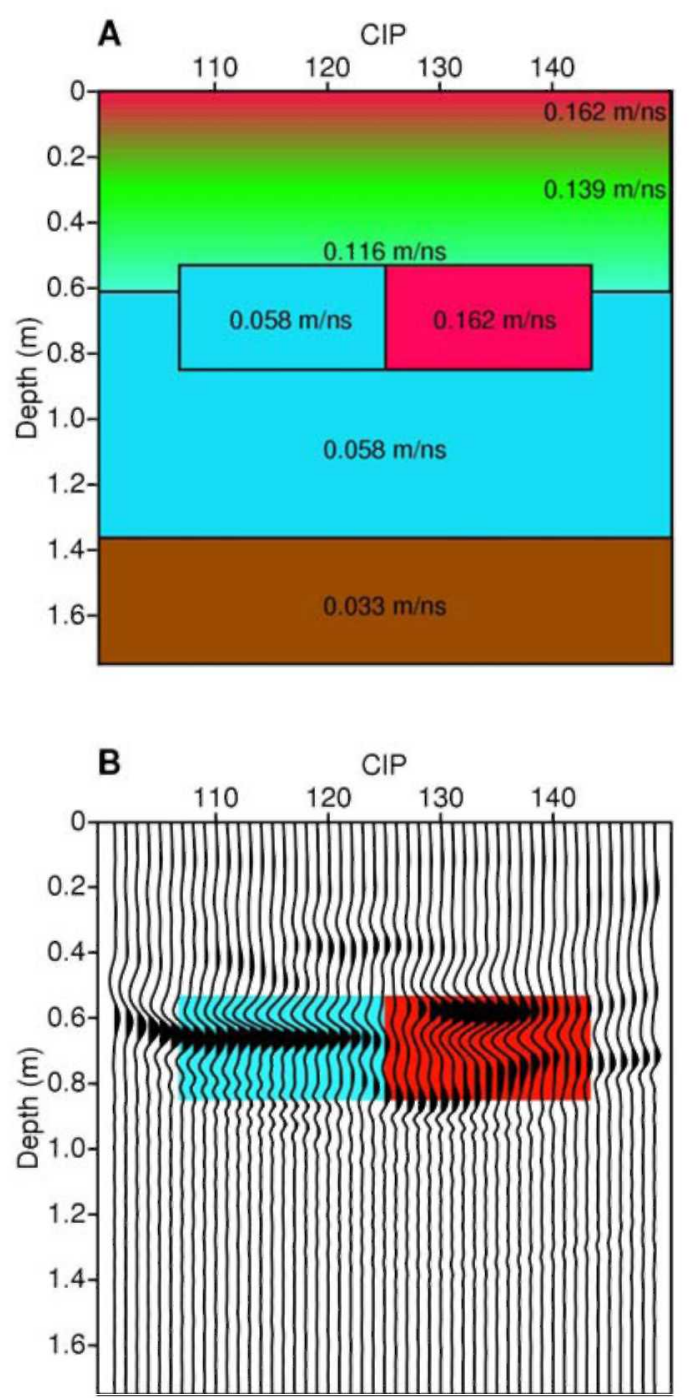

Figure 3. A) Velocity model, and B) Pre-stack depth migrated image of the containers filled with gasoline (red) and water (blue) saturated sand. Colored regions in the migrated image indicate actual positions and dimensions of the containers. The velocity gradient was derived from the surface velocity and effective interval velocity and was used to determine the angle-of-incidence at the top of the containers.

4.0. The ratio at the gasoline sand boundary is $\varepsilon_{2} / \varepsilon_{1}=(0.116$ $\mathrm{m} / \mathrm{ns} / 0.162 \mathrm{~m} / \mathrm{ns})^{2}=0.51$.

Amplitude variation is only an indirect function of offset so the amplitude analysis is done more directly in the amplitude vs angle-of-incidence (AVA) domain. I compute the offset to angle-of-incidence transform at the target reflector using ray tracing through the PSDM velocity model (Figure 3A). Comparing curved rays to straight rays, the lengths of the travel paths change very little, but the angle-of-incidence at the target changes significantly. For the experiment geometry, the straight ray assumption predicts a maximum angle of incidence of about $58^{\circ}$, but the linear gradient model predicts a maximum angle of incidence of only about $43^{\circ}$. This difference has a significant impact on predicted amplitudes and it is critical to use the curved ray incidence angle estimate.

\section{Amplitude pre-processing}

The data were prepared for amplitude and image processing by applying a bandpass filter (50-100-750-1200 MHz) to attenuate the low frequency transient present at small times, and to attenuate high frequency random noise. Additionally, I applied exponential gain, geometric spreading, and radiation pattern corrections. The exponential gain correction is based on an attenuation coefficient estimated in the following manner. First, the complex permittivity and magnetic permeability at $450 \mathrm{MHz}$ are calculated using the Cole-Cole equation for permittivity [8] and a similar equation for permeability. These values are then used to compute the complex wavenumber with the attenuation coefficient as the imaginary part of the wavenumber. Cole-cole parameters published by Powers and Olhoeft [9] give velocities at $450 \mathrm{MHz}$ that closely match the dry and water saturated pit sand $-0.158 \mathrm{~m} / \mathrm{ns}$ and $0.059 \mathrm{~m} / \mathrm{ns}$ respectively, so I assume that the tabulated parameters can be used to compute properties that approximate the pit sand. Further, using the parameters given by Powers and Olhoeft [9] to compute the attenuation coefficients for dry sand and moist sand yields values of 5.17 $\mathrm{dB} / \mathrm{m}$ and $5.30 \mathrm{~dB} / \mathrm{m}$ respectively. Since there is little difference between the dry and moist sands, I assume that the attenuation coefficient for moist sand will provide a reasonable estimate of the bulk attenuation above the containers. Using the effective interval velocity of $0.139 \mathrm{~m} / \mathrm{ns}$, the attenuation coefficient in the time domain is $0.737 \mathrm{~dB} / \mathrm{ns}$.

The take-off angle and angle-of-incidence for each offset is computed by ray tracing. The amplitude at each offset is then multiplied by the inverse of the radiation pattern coefficient for the corresponding take-off angle. In this case, I use the semiempirical radiation patterns defined by Bradford and Deeds [7].

\section{AVO analysis}

The peak frequency of the gasoline sand and water sand reflections is about $430 \mathrm{MHz}$ at near offsets and decreases to around $315 \mathrm{MHz}$ at the largest offset. The wavelength at 430 Mhz in the gasoline saturated sand is $0.38 \mathrm{~m}$ which is slightly greater than the thickness of the container $(0.30 \mathrm{~m})$. When the thickness of the layer is less than the signal wavelength, tuning effects may either increase or decrease the amplitude of the recorded wavelet depending on the product of vertical wavenumber and layer thickness. The problem becomes more

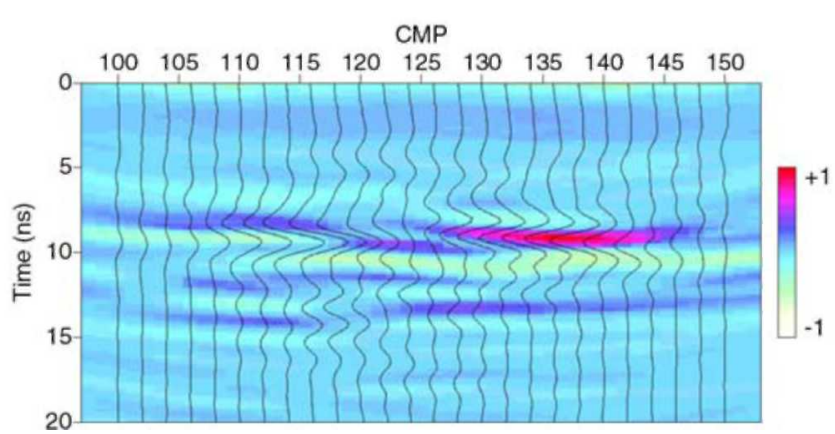

Figure 4. Relative PE AVA gradient image and the TE, pre-stack time migrated, wiggle trace overlay. The large AVA gradient associated with the top of the gasoline sand is clearly identifiable as a zone of red coincident with a high amplitude reflection. 

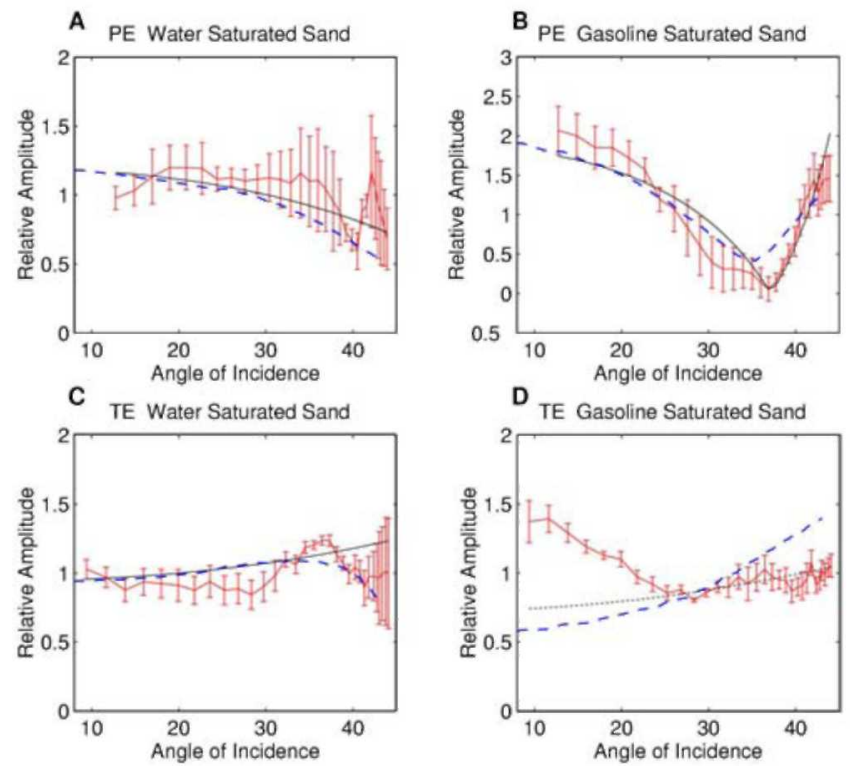

Figure 5. Data (red), Fresnel equation fit using a non-linear, least squares routine (black), and results of forward FDTD modeling using the velocity model in Figure 3 (dashed blue). Errorbars indicate one standard deviation. With the exception of D, the Fresnel and FDTD curves are a reasonable fit to the data.

complicated at far offsets where the resolution is lower due to decreasing vertical wavenumber with offset and lower frequency content due to frequency dependent attenuation. The width and length of the containers are also on the order of one wavelength and reverberation within the container may introduce additional tuning effects. The wavelength in the water saturated sand is about $0.13 \mathrm{~m}$ which is much smaller than the thickness of the container, and, with the exception of the side directly adjacent to the gasoline sand, the velocity contrast across the side and bottom boundaries is near zero.

For first pass interpretation, I apply a simple AVA gradient analysis by fitting the pre-stack time migrated reflection amplitudes with a $\sin ^{2} 9$ curve, then plot the gradient weighted by the zero offset intercept amplitude. This image then highlights reflections with a large gradient and large near offset reflection amplitude. The weighted gradient for the TM data is shown in Figure 4 with the stacked TE wiggle trace section overlain for comparison.

There are strong reflections associated with both the water and gasoline saturated sand, but there is a large value of the gradient for only the reflection from the gasoline sand (Figure 4). The image clearly indicates the reflection associated with a decrease in permittivity and allows for rapid qualitative interpretation.

Table 1. A comparison of permittivity ratios at the water sand and gasoline sand boundaries estimated from a non-linear fit of the reflection coefficient curves, Brewster's Angle analysis, and velocity analysis. The estimates from each method are in close agreement. Brewster's Angle was not reached for the water sand reflection. The Fresnel curve did not provide a reasonable fit to the TE gasoline reflection.

\begin{tabular}{|ccccc|}
\hline Interface & PE fit & TE fit & $\boldsymbol{\theta}_{\mathrm{B}}$ & Vel. analysis \\
\hline Moist Sand/Water Sand & 4.4 & 4.7 & -- & 4.0 \\
\hline Moist Sand/Gas Sand & .58 & -- & .56 & .51
\end{tabular}

Next, I used least squares, non-linear regression to fit Fresnel reflection coefficient curves to the top-of-container reflections. Amplitude curves for the analysis were taken from CMP supergathers calculated by summing adjacent CMP gathers along the water (CMPs 112-122) and gasoline (CMPs 132-142) reflectors. AVA curves are extracted from both the individual CMP gathers and from the supergathers. The amplitudes extracted from individual gathers are used to compute standard deviation in amplitude at each offset. Reflection coefficient curves are fit to the AVA curves extracted from the supergathers.

For the water sand reflection, curve fitting yields estimates in permittivity ratio $\left(\varepsilon_{2} / \varepsilon_{1}\right)$ of 4.10 and 4.17 for the PE and TE configurations respectively (Figure 5). The results are in good agreement with the permittivity ratio derived from velocity analysis (Table 1). For the gasoline saturated sand, analysis of the PE data gives a permittivity ratio of 0.58 (Figure 5) which is in good agreement with the value of 0.51 derived from velocity analysis (Table 1). The amplitude of the TE data decreases significantly with increasing offset (Figure 5). This is a major divergence from the expected result since the planewave reflection coefficients predict a positive AVA gradient. Finally, I forward modeled the data using an FDTD finite difference algorithm. The model is a full Maxwell equation simulator for lossy media. In this case I used line sources for both the TE and TM modes. I converted the velocity model derived through PSDM analysis (Figure 3) to a dielectric permittivity model. I used the Topp equation to estimate water content then used Archie's law with typical parameters for clean sand taken from the literature to estimate electric conductivity. The porosity is taken as the water content below the water table. Geometric spreading and attenuation corrections were applied as described above for the field data.

The numerical model produces a close match to the field data for all but the TE mode over the gas sand. Since the model does not include antenna radiation patterns, this gives confidence that our radiation pattern corrections to the field data were approximately correct. Some of the small scale deviations in the amplitude curves may be radiation pattern effects not included in the radiation correction or small scale heterogeneity not included in the model (e.g. between 35 and 45 degrees in Figure 5A). The FDTD model predicts a drop in amplitude at far offsets due to interference caused by scattering from the container boundaries. This drop is evident as a divergence from the Fresnel curves at far offset (Figure 5). These deviations from the Fresnel equations are caused by in line scattering at the container boundaries and appear to be present in the field data (e.g. Figure 5C).

The FDTD model did not predict a decreasing amplitude curve for the TE polarization over the gasoline saturated container as was observed in the field data. Conversely, the FDTD model amplitudes are close to the Fresnel equation but increase even more steeply. The difference between the field and predicted results in this one case is difficult to reconcile particularly given the good correlation observed in all other configurations. This may be due to a polarity dependent 3D tuning response, which would require full $3 \mathrm{D}$ wave equation 
modeling to properly analyze. Additional explanations include $3 \mathrm{D}$ heterogeneity not included in the $2 \mathrm{D}$ model or time dependent variability of the GPR system.

Brewster's Angle for the water sand reflection is not reached. However, $\theta_{\mathrm{B}}$ is clearly observed for the gasoline reflection (Figures 2 and 5) presenting the opportunity to use Brewster's Angle analysis. Data pre-processing consists only of bandpass filtering. Extracting the zero crossover angle from CMPs 133 - 140, I find that $\theta_{\mathrm{B}}$ occurs at an angle of 36.9 $\pm 1.6^{\circ}$ corresponding to $\mathrm{e}_{2} / \mathrm{e}_{1}=0.56 \pm 0.06$. The result differs from the velocity analysis estimate by only $8.4 \%$ and from the PE curve fitting result by only $3.6 \%$ (Table 1 ). This is a very encouraging result. Brewster's Angle analysis allows us to make a relatively precise estimate of the permittivity ratio at the moist sand/gasoline sand boundary with only the most minimal preprocessing.

\section{CONCLUSIONS}

The GPR AVO response is particularly sensitive to a decrease in permittivity across the reflecting boundary. Displacement of pore water with a wide variety of NAPLs can lead to zones of anomalously low permittivity making AVO analysis an attractive tool for direct detection of low permittivity contaminants.

Analyses of data from our physical model are surprising. Despite the three-dimensional, subwavelength character of the anomalies, the Fresnel equations largely describe the AVA curves and simple 2D forward wave equation modeling explains much of the deviation from the Fresnel equations. It is clear that acquiring data in multiple polarizations can substantially reduce uncertainty. While one certainly cannot draw broad generalizations from a single physical model, the results of this experiment suggest that it may be reasonable to make a number of simplifying assumptions in the analysis of field data that will make GPR AVO practical for contaminated site characterization.

\section{ACKNOWLEDGMENT}

This work was funded by the Houston Advanced Research Center and the U.S. Department of Energy, Environmental Management Science Program, Grant \# DE-FG07-99ER15008. James Loughridge helped with construction of the physical model and data acquisition.

\section{REFERENCES}

[1] X. Zeng, G. A. McMechan, and T. Xu, "Synthesis of amplitude versus offset variations in ground-penetrating radar data," Geophysics, vol. 65, pp. 113-125, 2000.

[2] M. L. Brewster and A. P. Annan, "Ground-penetrating radar monitoring of a controlled DNAPL release: $200 \mathrm{MHz}$ radar," Geophysics, vol. 59, pp. 1211-1221, 1994.

[3] J. J. Daniels, R. Robert, and M. Vendl, "Ground penetrating radar for the detection of liquid contaminants," J. Appl. Geophys., vol. 33, pp. 195207, 1995.

[4] T. Bergmann, J. O. A. Robertsson, and K. Holliger, "Finite-difference modeling of electromagnetic wave propagation in dispersive and attenuating media," Geophysics, vol. 63, pp. 856-867, 1998.

[5] P. M. Reppert, F. D. Morgan, and M. N. Toksoz, "Dielectric constant determination using ground-penetrating radar reflection coefficients," Journal of Applied Geophysics, vol. 43, pp. 189-197, 2000.

[6] G. S. Baker, "Applying AVO analysis to GPR data," Geophysical Research Letters, vol. 25, pp. 397-400, 1998.

[7] J. H. Bradford and J. C. Deeds, "Ground-penetrating radar theory and application of thinbed offset dependent reflectivity," Geophysics, vol. 71, pp. K47-K57, 2006.

[8] K. S. Cole and R. S. Cole, "Dispersion and absorption in dielectrics, I, alternating current characteristics," Journal of Chemical Physics, vol. 9, pp. 341-351, 1941.

[9] M. H. Powers and G. R. Olhoeft, "Modeling the response of leaking, buried pipes," presented at SAGEEP '95 Symposium on the Application of Geophysics to Environmental and Engineering Problems, Keystone, $\mathrm{CO}, 1996$. 